\title{
Generalized Heisenberg-Type Magnetic Phenomena in Coordination Polymers with Nickel- Lanthanide Dinuclear Units
}

Michał Antkowiak, ${ }^{[a, \S]}$ Mithun Chandra Majee, ${ }^{[b, \S]}$ Manoranjan Maity, ${ }^{[c, \$]}$ Dhrubajyoti Mondal, ${ }^{[\mathrm{d}]}$ Michalina Kaj, ${ }^{[e]}$ Monika Lesiów, ${ }^{[e]}$ Alina Bieńko, ${ }^{[e]}$ Leeor Kronik ${ }^{[f]}$, Muktimoy Chaudhury, ${ }^{[c]}$ Grzegorz Kamieniarz, ${ }^{[a, f]}$

[a] Faculty of Physics, A. Mickiewicz University, ul. Uniwersytetu Poznańskiego 2, 61-614 Poznań, Poland

[b] Banwarilal Bhalotia College, Kazi Nazrul University, Asansol, West Bengal-713303, India

[c] Department of Inorganic Chemistry, Indian Association for the Cultivation of Science, Jadavpur, Kolkata 700032 , India

[d] Department of Chemistry, Government General Degree College Mangalkote, University of Burdwan, West Bengal-713143, India

[e] Faculty of Chemistry, University of Wroclaw, 14 F. Joliot-Curie, 50-383 Wroclaw, Poland

[f] Department of Molecular Chemistry and Materials Science, Weizmann Institute of Science, Rehovoth 7610000, Israel.

[§] Michał Antkowiak, Mithun Chandra Majee, and Manoranjan Maity contributed equally in this work:

1. Figure S1. Partially labeled POV-Ray diagram for complex 2 S2

2. Figure S2. Partially labeled POV-Ray diagram for complex 4 S3

3. $\quad$ Figure S3. Partially labeled POV-Ray diagram for complex $5 \quad$ S4

4. Table S1. Selected bond distances $(\AA)$ and Angles (deg) for the Ni-Ln complexes $2-5$ ( $\mathrm{Ln}=\mathrm{Gd}, \mathrm{Tb}$, Dy, Ho, respectively) S5

5. Table S2. Results of Continuous SHAPE Measurement for complexes $\mathbf{2}$ - $\mathbf{5}$.

6. $\quad$ Figure S4. MIR and FIR spectra for complexes 1-5

7. Figure S5. Thermal dependence of the inverse magnetic susceptibility for compounds $\mathbf{1}-\mathbf{5}$. 


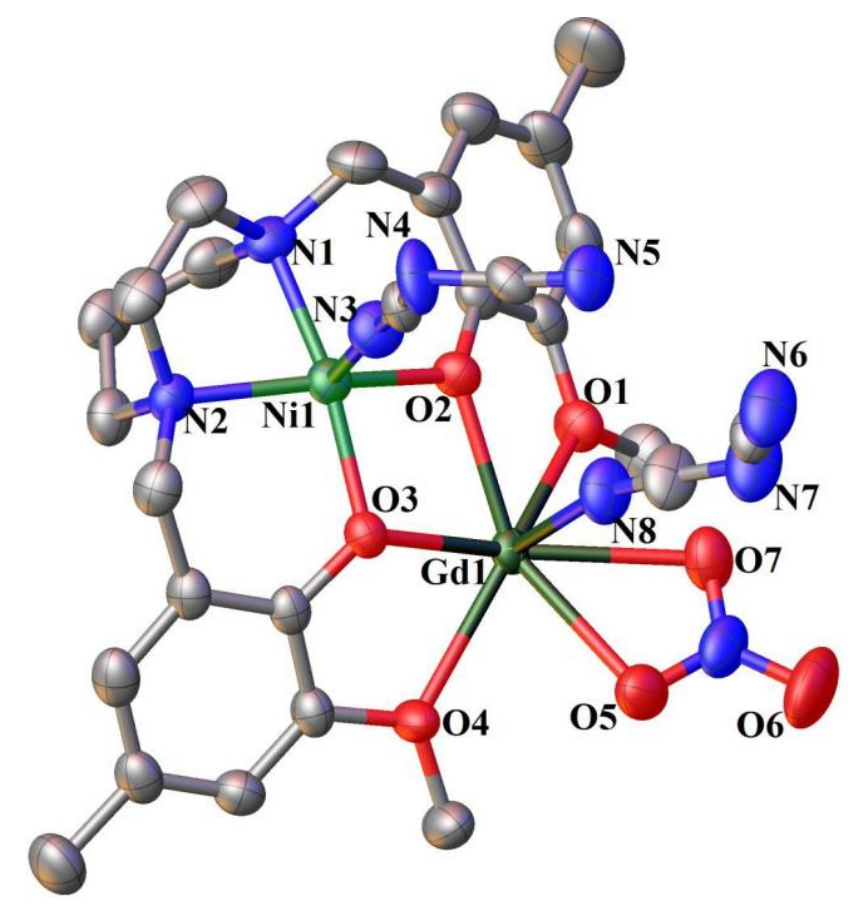

Figure S1. Partially labeled POV-Ray (in ellipsoid form) diagram showing the atom-labeling scheme in the heterobimetallic fragment $\mathrm{Ni}^{\mathrm{II}}-\mathrm{Gd}^{\mathrm{III}}$ of complex 2. 


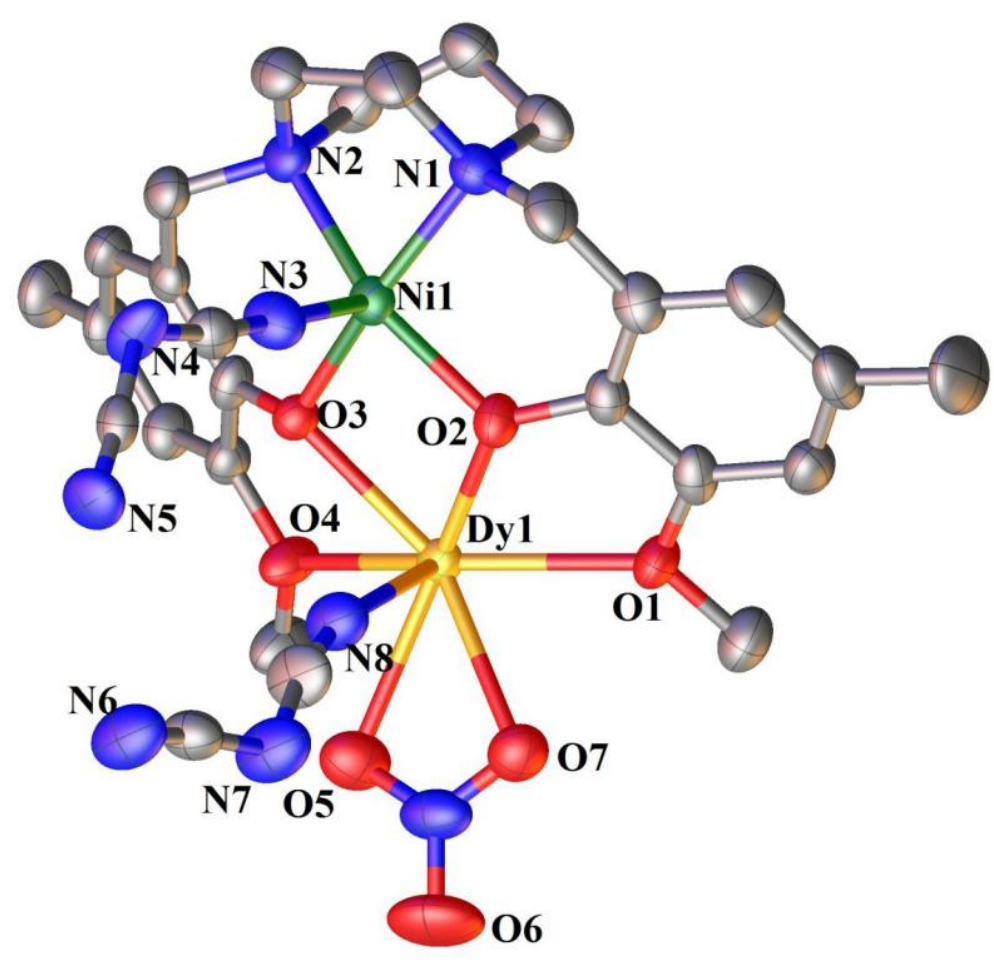

Figure S2. Partially labeled POV-Ray (in ellipsoid form) diagram showing the atom-labeling scheme in the $\mathrm{Ni}^{\mathrm{II}}-\mathrm{Dy}{ }^{\mathrm{III}}$ moiety of complex 4. 


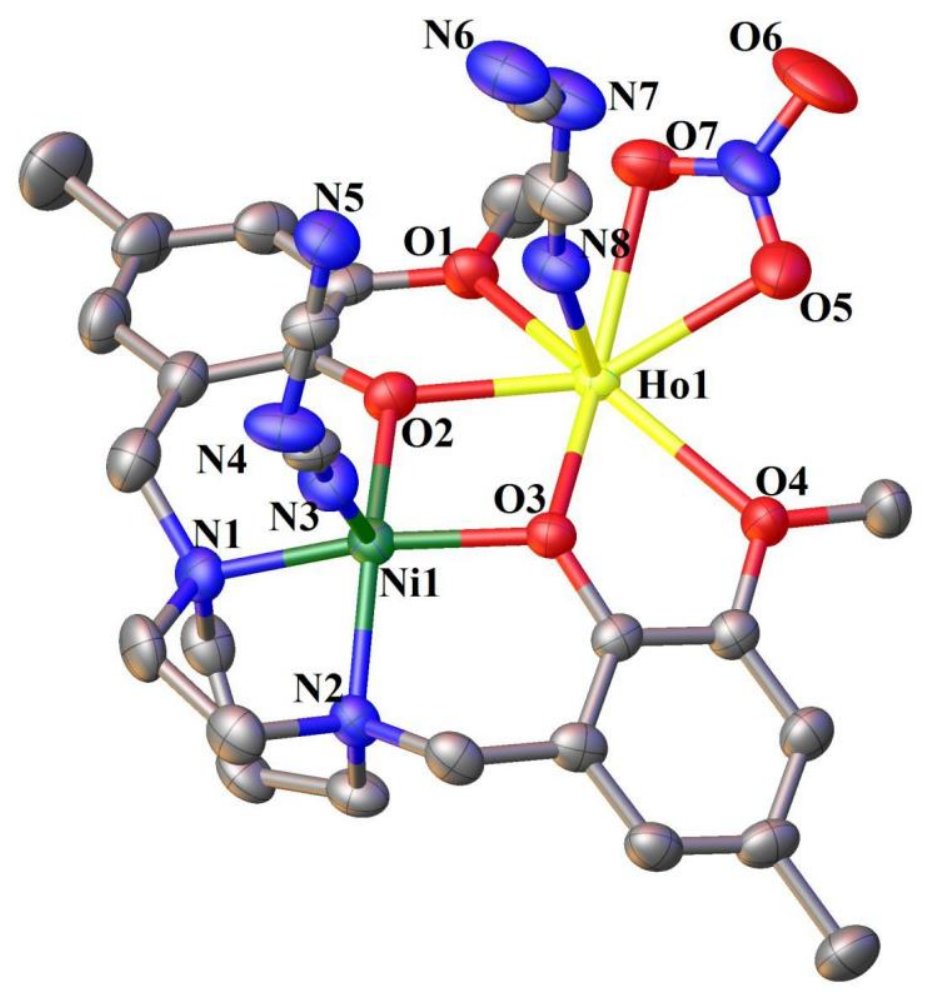

Figure S3. Partially labeled POV-Ray (in ellipsoid form) diagram showing the atom-labeling scheme in the $\mathrm{Ni}^{\mathrm{II}}-\mathrm{Ho}^{\mathrm{III}}$ bimetallic unit of complex 5. 
Table S1. Selected bond distances $(\AA)$ and Angles (deg) for the Ni-Ln complexes $2-\mathbf{5}(\mathrm{Ln}=\mathrm{Gd}, \mathrm{Tb}$, Dy, Ho, respectively).

\begin{tabular}{|c|c|c|c|c|}
\hline \multicolumn{5}{|c|}{ Bond distances $(\AA)$} \\
\hline Parameter & 2 & 3 & 4 & 5 \\
\hline $\mathrm{Ln} 1-\mathrm{O} 3$ & $2.325(4)$ & $2.302(3)$ & $2.331(5)$ & $2.321(3)$ \\
\hline $\mathrm{Ln} 1-\mathrm{O} 2$ & $2.364(4)$ & $2.348(3)$ & $2.292(6)$ & $2.279(3)$ \\
\hline Ln1-N5 & $2.478(5)$ & $2.462(4)$ & $2.453(8)$ & $2.423(4)$ \\
\hline $\mathrm{Ln} 1-\mathrm{N} 8$ & $2.511(5)$ & $2.490(5)$ & $2.482(8)$ & $2.470(4)$ \\
\hline $\mathrm{Ln} 1-\mathrm{O} 7$ & $2.513(5)$ & $2.494(4)$ & $2.485(6)$ & $2.477(3)$ \\
\hline $\mathrm{Ln} 1-\mathrm{O} 5$ & $2.517(4)$ & $2.494(3)$ & $2.483(7)$ & $2.471(4)$ \\
\hline $\mathrm{Ln} 1-\mathrm{O} 1$ & $2.517(4)$ & $2.516(3)$ & $2.550(5)$ & $2.542(3)$ \\
\hline Ln1-N6 & $2.523(5)$ & $2.504(5)$ & $2.499(8)$ & $2.482(4)$ \\
\hline $\mathrm{Ln} 1-\mathrm{O} 4$ & $2.551(4)$ & $2.563(3)$ & $2.514(6)$ & $2.497(3)$ \\
\hline $\mathrm{Ni1}-\mathrm{O} 3$ & $1.973(4)$ & $1.989(3)$ & $1.992(5)$ & $1.991(3)$ \\
\hline Ni1-N3 & $1.974(5)$ & $1.972(4)$ & $1.978(8)$ & $1.980(4)$ \\
\hline $\mathrm{Ni1}-\mathrm{O} 2$ & $1.992(3)$ & $1.997(3)$ & $1.981(5)$ & $1.980(3)$ \\
\hline $\mathrm{Ni} 1-\mathrm{N} 2$ & $2.046(4)$ & $2.039(4)$ & $2.039(7)$ & $2.042(4)$ \\
\hline $\mathrm{Ni1}-\mathrm{N} 1$ & $2.048(4)$ & $2.051(4)$ & $2.045(7)$ & $2.034(4)$ \\
\hline \multicolumn{5}{|c|}{ Bond angles (deg) } \\
\hline $\mathrm{O} 3-\mathrm{Ln} 1-\mathrm{O} 2$ & $69.27(12)$ & $69.95(11)$ & $70.02(18)$ & $70.22(11)$ \\
\hline $\mathrm{O} 3-\mathrm{Ln} 1-\mathrm{N} 5$ & $75.05(15)$ & $75.39(14)$ & $73.7(2)$ & $74.07(14)$ \\
\hline $\mathrm{O} 2-\mathrm{Ln} 1-\mathrm{N} 5$ & $73.87(14)$ & $73.97(13)$ & $75.6(2)$ & $75.37(14)$ \\
\hline $\mathrm{O} 3-\mathrm{Ln} 1-\mathrm{N} 8$ & $78.00(16)$ & 77.93(16) & $79.2(2)$ & $79.13(13)$ \\
\hline $\mathrm{O} 2-\mathrm{Ln} 1-\mathrm{N} 8$ & $79.47(15)$ & $79.53(14)$ & $77.3(2)$ & $77.41(15)$ \\
\hline $\mathrm{N} 5-\mathrm{Ln} 1-\mathrm{N} 8$ & $147.26(17)$ & $147.39(16)$ & $146.7(2)$ & $146.82(16)$ \\
\hline $\mathrm{O} 3-\mathrm{Ln} 1-\mathrm{O} 7$ & $147.39(15)$ & $147.33(14)$ & $149.33(19)$ & $149.24(11)$ \\
\hline $\mathrm{O} 2-\mathrm{Ln} 1-\mathrm{O} 7$ & $113.32(15)$ & $113.11(12)$ & $108.6(2)$ & $108.69(13)$ \\
\hline $\mathrm{N} 5-\mathrm{Ln} 1-\mathrm{O} 7$ & $137.56(16)$ & $137.28(15)$ & $136.7(2)$ & $136.42(13)$ \\
\hline $\mathrm{N} 8-\mathrm{Ln} 1-\mathrm{O} 7$ & $70.97(18)$ & $71.10(17)$ & $70.9(2)$ & $70.91(14)$ \\
\hline $\mathrm{O} 3-\mathrm{Ln} 1-\mathrm{O} 5$ & $109.75(14)$ & $109.13(13)$ & $112.7(2)$ & $112.38(12)$ \\
\hline $\mathrm{O} 2-\mathrm{Ln} 1-\mathrm{O} 5$ & $149.45(13)$ & $149.38(12)$ & $146.7(2)$ & $146.99(13)$ \\
\hline N5-Ln1-O5 & $136.43(15)$ & $136.42(14)$ & $137.7(2)$ & $137.64(14)$ \\
\hline $\mathrm{N} 8-\mathrm{Ln} 1-\mathrm{O} 5$ & $70.76(16)$ & $70.64(15)$ & $71.0(3)$ & $71.17(16)$ \\
\hline $\mathrm{O} 7-\mathrm{Ln} 1-\mathrm{O} 5$ & $50.54(15)$ & $50.76(13)$ & $51.2(2)$ & $51.39(13)$ \\
\hline $\mathrm{O} 3-\mathrm{Ln} 1-\mathrm{O} 1$ & $130.77(12)$ & $131.72(10)$ & $131.50(18)$ & $131.95(10)$ \\
\hline $\mathrm{O} 2-\mathrm{Ln} 1-\mathrm{O} 1$ & $63.87(13)$ & $64.14(11)$ & $64.25(18)$ & $64.30(11)$ \\
\hline $\mathrm{N} 5-\mathrm{Ln} 1-\mathrm{O} 1$ & $78.34(15)$ & $78.40(14)$ & $80.2(2)$ & $80.23(13)$ \\
\hline $\mathrm{N} 8-\mathrm{Ln} 1-\mathrm{O} 1$ & $106.60(16)$ & $106.75(15)$ & $104.9(2)$ & $104.52(14)$ \\
\hline $\mathrm{O} 7-\mathrm{Ln} 1-\mathrm{O} 1$ & $69.65(14)$ & $69.17(12)$ & $65.2(2)$ & $64.78(12)$ \\
\hline $\mathrm{O} 5-\mathrm{Ln} 1-\mathrm{O} 1$ & $118.08(14)$ & $117.81(12)$ & $114.2(2)$ & $114.00(12)$ \\
\hline $\mathrm{O} 3-\mathrm{Ln} 1-\mathrm{N} 6$ & $129.89(15)$ & 129.94(14) & $128.9(2)$ & $129.09(12)$ \\
\hline $\mathrm{O} 2-\mathrm{Ln} 1-\mathrm{N} 6$ & $128.94(14)$ & $128.88(13)$ & $130.1(2)$ & $129.82(13)$ \\
\hline N5-Ln1-N6 & $69.51(17)$ & $69.60(15)$ & $69.8(2)$ & $69.76(14)$ \\
\hline N8- $\mathrm{Ln} 1-\mathrm{N} 6$ & $143.23(18)$ & 143.01(16) & $143.5(3)$ & $143.41(15)$ \\
\hline O5- Ln1-N6 & $76.42(16)$ & $76.42(16)$ & $75.9(3)$ & $75.80(15)$ \\
\hline $\mathrm{O} 1-\mathrm{Ln} 1-\mathrm{N} 6$ & $74.72(15)$ & $74.25(13)$ & $75.1(2)$ & $74.99(13)$ \\
\hline $\mathrm{O} 3-\mathrm{Ln} 1-\mathrm{O} 4$ & $63.89(12)$ & $63.90(11)$ & $64.26(19)$ & $64.33(11)$ \\
\hline $\mathrm{O} 2-\mathrm{Ln} 1-\mathrm{O} 4$ & $130.69(12)$ & $131.37(10)$ & $131.94(18)$ & $132.09(10)$ \\
\hline $\mathrm{N} 5-\mathrm{Ln} 1-\mathrm{O} 4$ & $79.99(15)$ & $80.33(13)$ & $78.1(2)$ & $78.41(13)$ \\
\hline $\mathrm{N} 8-\mathrm{Ln} 1-\mathrm{O} 4$ & $104.37(16)$ & $104.29(15)$ & $107.0(2)$ & $107.16(14)$ \\
\hline $\mathrm{O} 7-\mathrm{Ln} 1-\mathrm{O} 4$ & $114.23(14)$ & $113.83(12)$ & $118.1(2)$ & $117.94(12)$ \\
\hline $\mathrm{O} 5-\mathrm{Ln} 1-\mathrm{O} 4$ & $65.71(14)$ & $65.19(12)$ & $69.2(2)$ & $68.89(11)$ \\
\hline $\mathrm{O} 1-\mathrm{Ln} 1-\mathrm{O} 4$ & $148.03(12)$ & 147.71(11) & $146.92(19)$ & $146.99(11)$ \\
\hline N6-Ln1-O4 & $75.83(15)$ & $75.69(13)$ & $74.1(2)$ & $74.14(13)$ \\
\hline $\mathrm{O} 3-\mathrm{Ni} 1-\mathrm{N} 3$ & $105.45(19)$ & $105.23(17)$ & $100.1(3)$ & $100.46(15)$ \\
\hline $\mathrm{O} 3-\mathrm{Ni} 1-\mathrm{O} 2$ & $84.48(15)$ & $83.97(13)$ & $83.8(2)$ & $83.58(13)$ \\
\hline $\mathrm{N} 3-\mathrm{Ni} 1-\mathrm{O} 2$ & $100.36(17)$ & $100.01(16)$ & $104.9(3)$ & $105.40(16)$ \\
\hline $\mathrm{O} 3-\mathrm{Ni} 1-\mathrm{N} 2$ & $93.85(18)$ & $94.33(15)$ & $96.2(2)$ & $95.52(11)$ \\
\hline $\mathrm{N} 3-\mathrm{Ni} 1-\mathrm{N} 2$ & $96.50(19)$ & $96.67(17)$ & $97.5(3)$ & $96.94(16)$ \\
\hline $\mathrm{O} 2-\mathrm{Ni} 1-\mathrm{N} 2$ & $162.89(15)$ & $163.09(17)$ & $157.3(3)$ & $157.29(14)$ \\
\hline $\mathrm{O} 3-\mathrm{Ni} 1-\mathrm{N} 1$ & 156.81(16) & 157.22(16) & $162.7(2)$ & $162.96(13)$ \\
\hline $\mathrm{N} 3-\mathrm{Ni} 1-\mathrm{N} 1$ & $97.32(19)$ & $97.19(17)$ & $97.0(3)$ & $96.42(17)$ \\
\hline $\mathrm{O} 2-\mathrm{Ni} 1-\mathrm{N} 1$ & $95.84(16)$ & $96.20(14)$ & $94.0(3)$ & $94.18(15)$ \\
\hline $\mathrm{N} 2-\mathrm{Ni} 1-\mathrm{N} 1$ & $79.06(19)$ & $78.90(16)$ & $79.2(3)$ & $79.07(15)$ \\
\hline
\end{tabular}


Table S2. Results of Continuous SHAPE Measurement (CShM) analysis of the 13 possible coordination geometries with nine coordination number of Lanthanide (III) ions for the complexes 2 -

5.

\begin{tabular}{|l|l|l|l|l|l|}
\hline \multicolumn{1}{|c|}{ Geometry } & Symmetry & Complex 2 & Complex 3 & Complex 4 & Complex 5 \\
\hline Enneagon = EP-9 & D9h & $33.784(4)$ & $33.820(3)$ & $33.980(3)$ & $34.021(5)$ \\
\hline Octagonal pyramid = OPY-9 & C8v & $22.693(7)$ & $22.754(5)$ & $22.697(4)$ & $22.785(5)$ \\
\hline Heptagonal bipyramid = HBPY-9 & D7h & $14.721(4)$ & $14.608(8)$ & $14.607(4)$ & $14.594(7)$ \\
\hline Johnson triangular cupola J3 = JTC-9 & C3v & $15.337(3)$ & $15.355(2)$ & $15.348(6)$ & $15.317(4)$ \\
\hline Capped cube J8 = JCCU-9 & C4v & $9.666(2)$ & $9.638(5)$ & $9.689(6)$ & $9.757(6)$ \\
\hline $\begin{array}{l}\text { Spherical-relaxed capped cube }= \\
\text { CCU-9 }\end{array}$ & C4v & $7.870(3)$ & $7.880(5)$ & $7.949(3)$ & $7.878(2)$ \\
\hline $\begin{array}{l}\text { Capped square antiprism J10 = } \\
\text { JCSAPR-9 }\end{array}$ & C4v & $3.969(2)$ & $3.906(4)$ & $3.772(5)$ & $3.777(6)$ \\
\hline $\begin{array}{l}\text { Spherical capped square antiprism }= \\
\text { CSAPR-9 }\end{array}$ & C4v & $3.071(7)$ & $3.055(4)$ & $2.936(3)$ & $2.944(4)$ \\
\hline $\begin{array}{l}\text { Tricappedtrigonal prism J51 = } \\
\text { JTCTPR-9 }\end{array}$ & D3h & $5.568(2)$ & $5.553(5)$ & $5.439(2)$ & $5.496(6)$ \\
\hline $\begin{array}{l}\text { Spherical tricappedtrigonal prism }= \\
\text { TCTPR-9 }\end{array}$ & D3h & $3.684(5)$ & $3.676(3)$ & $3.564(7)$ & $3.540(2)$ \\
\hline $\begin{array}{l}\text { Tridiminished icosahedron J63 }= \\
\text { JTDIC-9 }\end{array}$ & C3v & $13.385(2)$ & $13.446(4)$ & $13.441(8)$ & $13.517(8)$ \\
\hline Hula-hoop = HH-9 & C2v & $6.276(4)$ & $6.323(8)$ & $6.534(9)$ & $6.480(2)$ \\
\hline Muffin = MFF-9 & Cs & $2.307(2)$ & $2.258(8)$ & $2.168(2)$ & $2.161(5)$ \\
\hline
\end{tabular}

The minimum value indicated in bold

CShM values between 0.1 and 3 usually correspond to a not negligible but still small distortion from ideal geometry.

(a) M. Llunell, D. Casanova, J. Cicera, P. Alemany and S. Alvarez, SHAPE, Version 2.1, Barcelona, Spain, 2013; (b) Alvarez, S.; Alemany, P.; Casanova, D.; Cirera, J.; Llunell, M.; Avnir, D. Shape maps and polyhedral interconversion paths in transition metal chemistry. Coord. Chem. Rev. 2005, 249, 1693-1708 

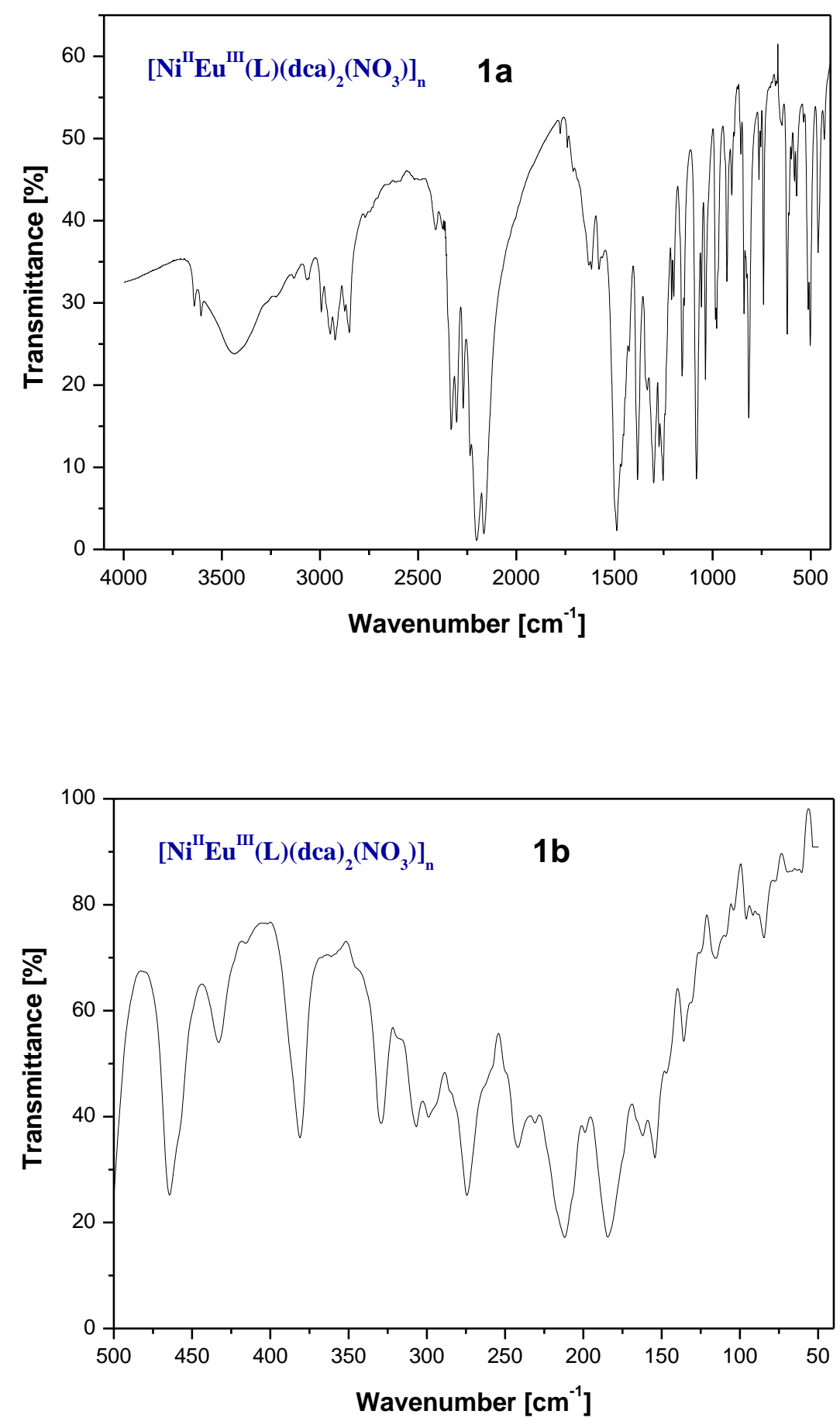

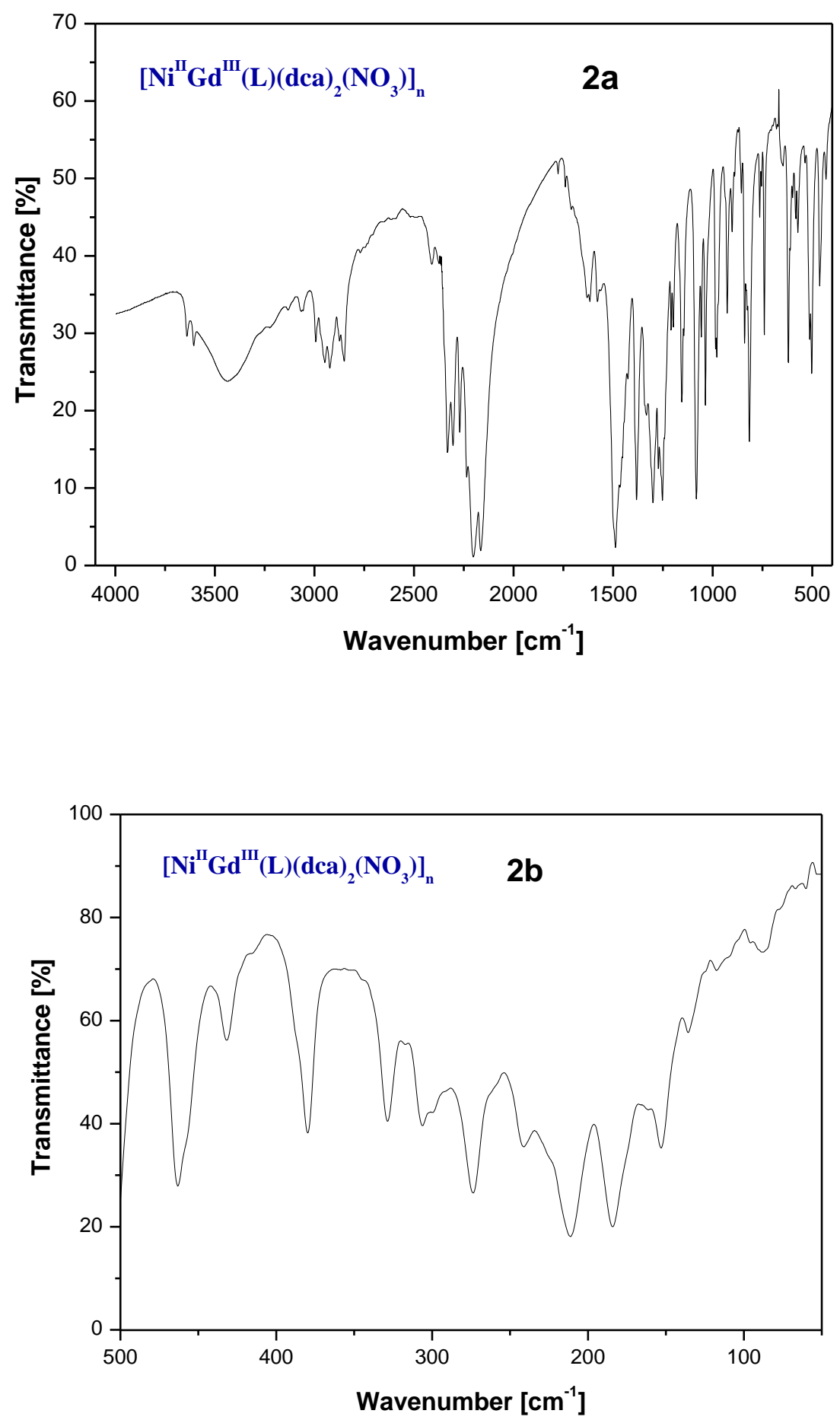

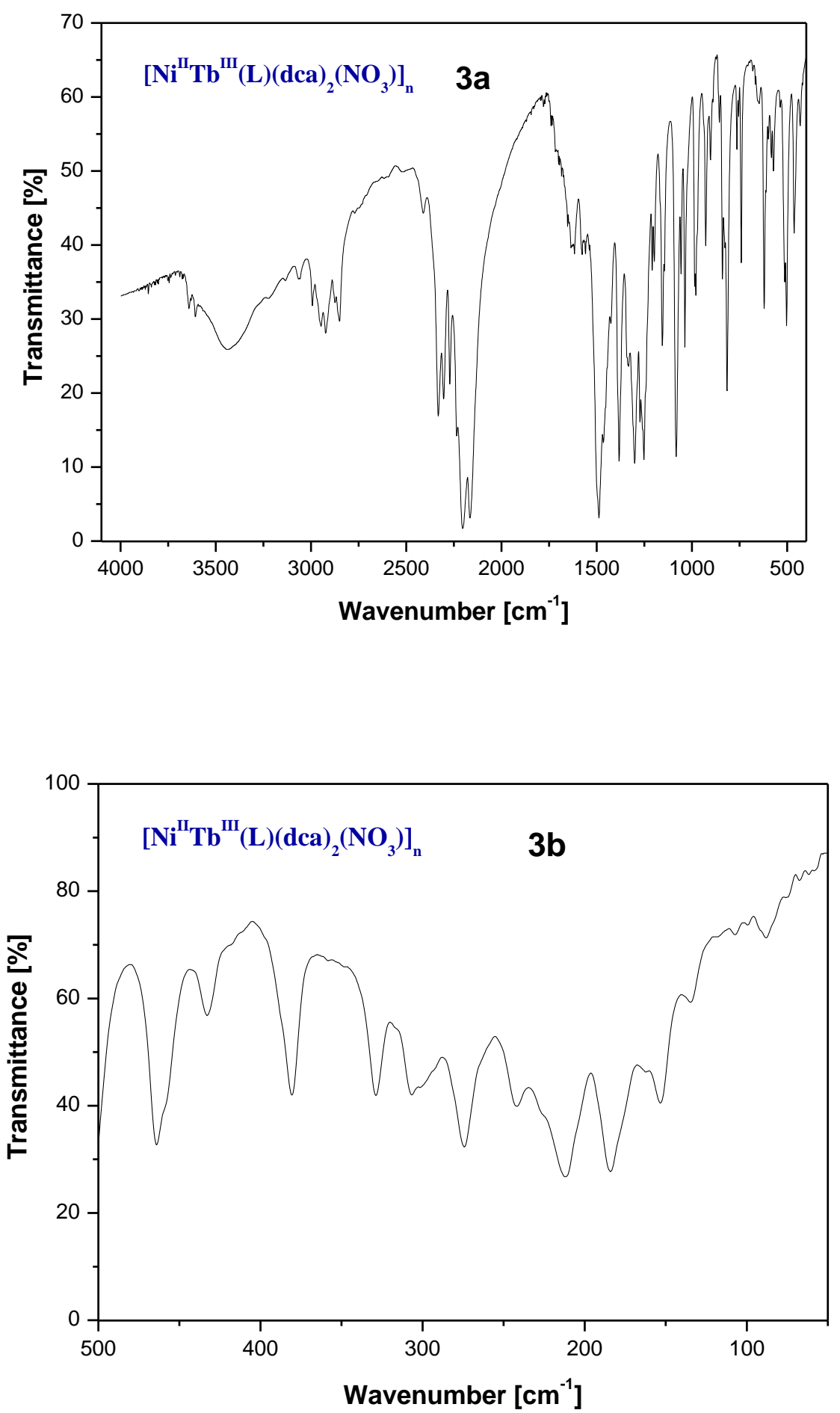

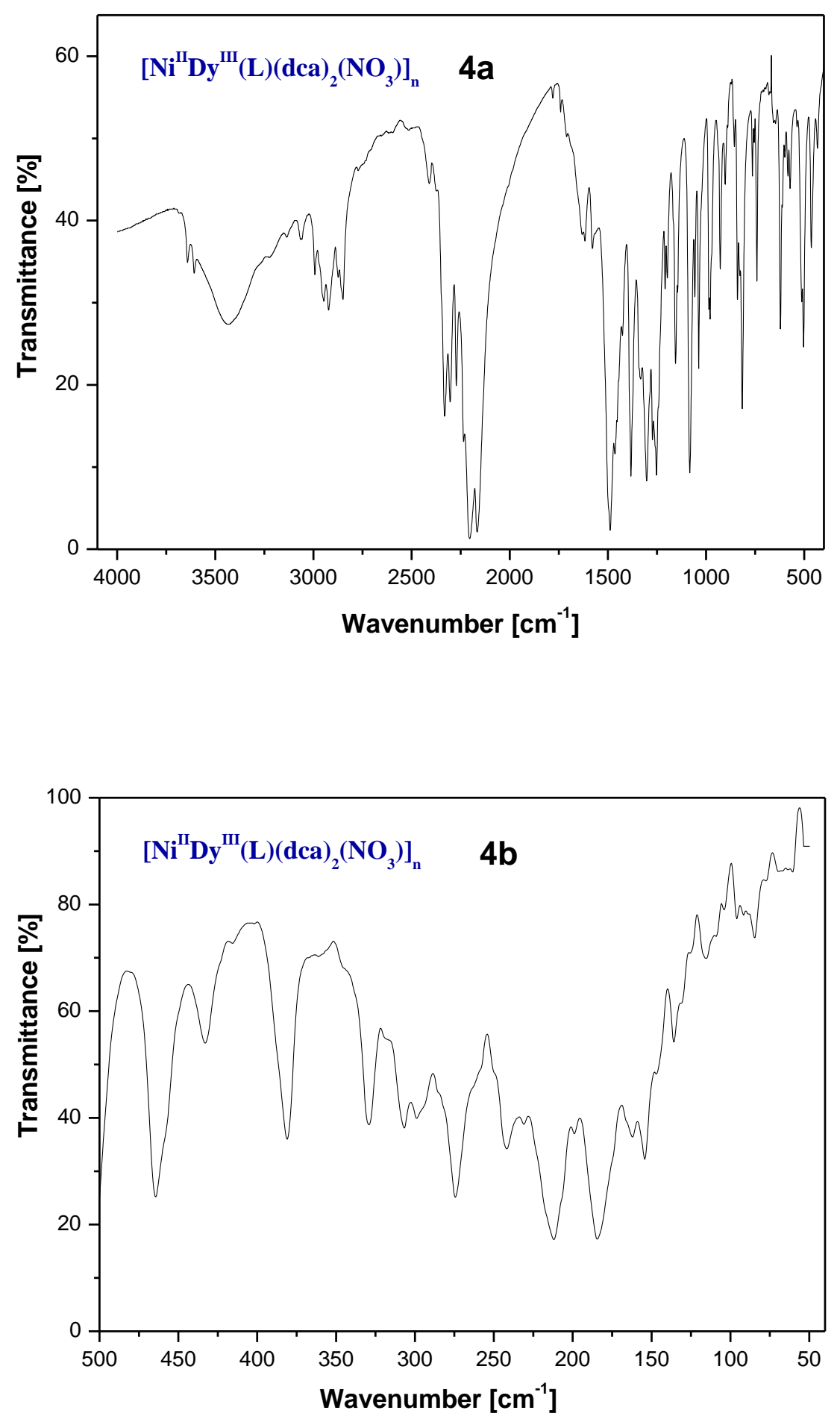

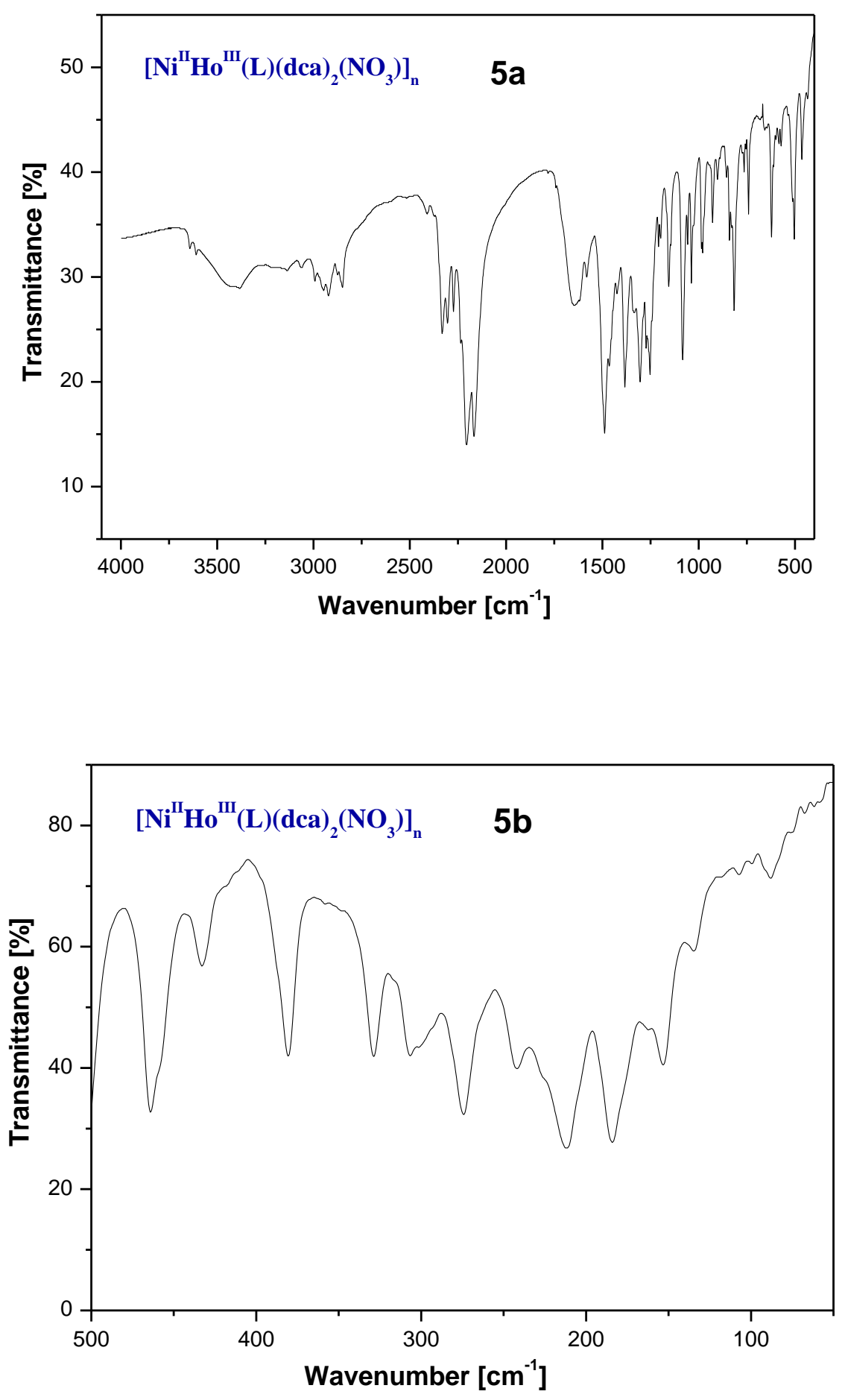

Figure S4. MIR and FIR spectra for complexes $1-\mathbf{5}$. The legend provides the chemical formulae of the compounds, together with two-character labels indicating the number of a given compound and the type of measurement, namely "a" for MIR and "b" for FIR. 


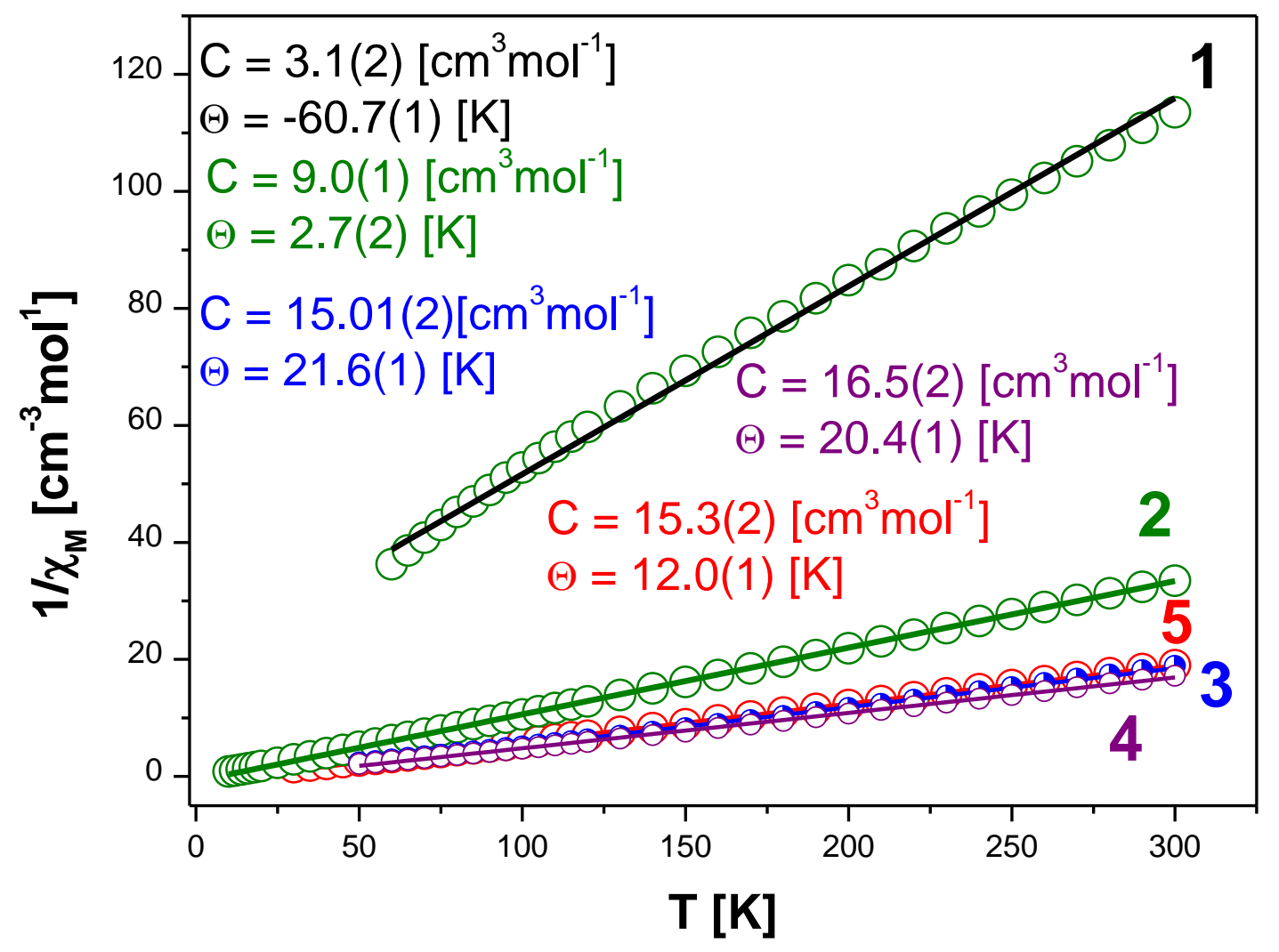

Figure. S5. Thermal dependence of the inverse magnetic susceptibility for compounds $\mathbf{1}-\mathbf{5}$. 